\title{
Improving endoscopy efficiency by reducing turnaround time between cases
}

\author{
Authors: Kathleen Bryce, Robert Fearn and Sam Murray
}

\section{Aims}

An improvement in the efficiency of endoscopy lists could help meet increasing demand. We aimed to reduce the 'turnaround time' between procedures.

\section{Methods}

An endoscopy quality improvement fellow coordinated efficiency projects. Timings were collected from the electronic patient record, from entries made by endoscopy staff (time of patient entry into endoscopy room, procedure start, finish, and time out of room) and entered into a spreadsheet. For in-depth assessment of activities during turnaround, the fellow observed the whole list, collecting information in real time. Baseline results were discussed at the departmental meeting. Suggestions for improvement included nurse training in consent/cannulation and creation of a 'turnaround nurse' (TN) who would minimise turnaround time by performing those tasks necessary between cases. We re-measured to see the effect.

\section{Results}

A preliminary audit of 94 turnaround times over 5 days ( 23 lists) showed an average turnaround time of 20.3 minutes. We excluded instances where the next patient did not attend / was cancelled.

Two lists were directly observed. The activities performed simultaneously by the endoscopist, trainee and nurses included 20 minutes consenting per list, 7.5 minutes cannulating, 47 minutes typing reports, 12.5 minutes handling histology specimens, 47.5 minutes for equipment turnaround and 22 minutes for patient transfer/handover.

The impact of the TN was then assessed over 5 days. Twenty lists (75 turnarounds) were examined without TN (control): turnaround time was still 20.8 minutes (range $6-57$, median 19). Where the TN was present (three lists, 12 turnarounds), average turnaround was 18.4 minutes (range 5-42, median 16).

Two lists were assessed in detail. In contrast to the previous audit, no time was spent by the endoscopist on consent/ cannulation during turnaround when the TN was present. Time taken on other activities remained the same.

Authors: Gastroenterology, Homerton University Hospital NHS Foundation Trust, London, UK

\section{Conclusion}

The implementation of a TN has removed the need for consent and cannulation during turnaround, freeing up the endoscopist for more value-added tasks. Average turnaround time only decreased by 2 minutes, but could save 20 minutes over a 12-point list. This is likely because there are still unresolved obstructions to flow of the patients through the unit. There were patient factors (lateness, delay in getting ready), cases when the endoscope was not ready, and delay in typing the electronic report. We plan to train the nursing staff to help set up the computer/report and address the other issues in future cycles of the improvement project.

\section{Conflict of interest statement}

None declared. 